\title{
Comorbidity in US patients with multiple sclerosis
}

This article was published in the following Dove Press journal: Patient Related Outcome Measures

\section{Natalie C Edwards' \\ Michael Munsell ${ }^{2}$ \\ Joseph Menzin ${ }^{2}$ \\ Amy L Phillips ${ }^{3}$}

'Health Services Consulting Corporation (NCE), Boxborough, MA, USA; ${ }^{2}$ Boston Health Economics, Inc., Waltham, MA, USA; ${ }^{3}$ EMD Serono, Inc., Rockland, MA, USA
Correspondence: Natalie C Edwards Health Services Consulting Corporation, 169 Summer Road, Boxborough, MA 01719 , USA

$\mathrm{Tel}+\mathrm{I} 9782640712$

Fax + I $97826407 \mid 3$

Email nedwards.hscc@gmail.com
Objective: To assess the trends in the prevalence of comorbidities in US patients with multiple sclerosis (MS), and the association of demographic characteristics with the presence of comorbidities.

Study design: A retrospective analysis was conducted from a sample of 5 million patients from the IMS Health Real World Data Adjudicated Claims - US database.

Methods: Comorbidity in patients with MS was assessed by year (2006-2014), and logistic regression models evaluated the association of age, sex, and region with select comorbidities.

Results: The most common comorbidities from 2006 to 2014 were hyperlipidemia and hypertension $(25.9 \%-29.7 \%$ of patients within an individual year), followed by gastrointestinal disease (18.4\%-21.2\% of patients) and thyroid disease $(12.9 \%-17.1 \%$ of patients). The proportion with a claim for hyperlipidemia increased from 2006 to 2009, was stable from 2009 to 2011, and then declined from 2011 to 2014. The proportion with a claim for hypertension generally increased from 2006 to 2013, then declined from 2013 to 2014. The proportion with a claim for gastrointestinal disease, thyroid disease, and anxiety generally increased from 2006 to 2014 . Claims for comorbidities were statistically significantly more likely among older age groups $(p<0.05)$, with the exception of anxiety and alcohol abuse, which were statistically significantly less likely among older age groups. Claims for gastrointestinal disease $(\mathrm{OR}=0.75)$, thyroid disease $(\mathrm{OR}=0.36)$, chronic lung disease $(\mathrm{OR}=0.76)$, arthritis $(\mathrm{OR}=0.71)$, anxiety $(\mathrm{OR}=0.63)$, and depression $(\mathrm{OR}=0.69)$ were statistically significantly less likely among males versus females (all $p<0.05)$. Claims for hyperlipidemia $(\mathrm{OR}=1.39)$, hypertension $(\mathrm{OR}=1.25)$, diabetes $(\mathrm{OR}=1.31)$, and alcohol abuse $(\mathrm{OR}=2.41)$ were significantly more likely among males $(p<0.05)$. Many comorbidity claims were statistically significantly more likely in the Northeast and South compared with the Midwest and West.

Conclusion: This study provides select comorbidity claims estimates in US patients with MS, and thus highlights the importance of comprehensive patient care approaches.

Keywords: multiple sclerosis, prevalence, comorbidities, disease-modifying drugs

\section{Plain language summary}

Comorbidities are other medical conditions present in patients with an illness. Comorbidities in patients with multiple sclerosis (MS) can delay MS diagnosis, increase disability progression, reduce quality of life, increase hospitalization, and increase the chance of death. This study assessed comorbidity diagnoses in US patients with MS from 2006-2014, and evaluated if comorbidities differed by patient sex, age, and geographic region. Diagnoses of high cholesterol, high blood pressure, gastrointestinal disorders, thyroid disease, and anxiety were common with MS. The percentage of patients with MS with gastrointestinal disorders, thyroid disease, 
and anxiety increased over the 9 years. The percentage with high cholesterol increased from 2006-2009, was stable from 2009-2011, then declined from 2011-2014. The percentage with hypertension increased from 2006-2013, then declined from 2013-2014. Comorbidities were more common among older patients, except for anxiety, depression, and alcohol abuse. Diagnoses of gastrointestinal disease, lung disease, arthritis, anxiety, and depression were more common among women. High cholesterol, high blood pressure, diabetes, and alcohol abuse were more common among men. More patients in the Northeast and South had comorbidities compared with patients in the Midwest and West. A better understanding of comorbidities in MS may improve patient support, health care services, and quality of life.

\section{Introduction}

Multiple sclerosis (MS) is a chronic, recurrent, inflammatory, demyelinating disease of the central nervous system. ${ }^{1}$ An estimated 400,000 people in the US have $\mathrm{MS}^{2,3}$ and more than 2 million people live with the disease worldwide. ${ }^{4}$ The impact of comorbidities in MS is an important and increasing area of interest. ${ }^{5}$ Comorbid conditions are common in patients with MS, and can lead to poorer outcomes. ${ }^{6}$ Emerging evidence has shown that the presence of comorbidities may delay MS diagnosis, ${ }^{7}$ increase the rate of disability progression, ${ }^{8}$ reduce quality of life, ${ }^{9,10}$ increase rates of hospitalization, ${ }^{11}$ and increase the hazard of death. ${ }^{12}$

The reported prevalence of comorbidities in patients with MS varies widely ${ }^{13}$ depending on the range of comorbid conditions considered, the specific patient population evaluated, and factors such as sex, age, race, geographic region, lifestyle factors, and socioeconomic status. ${ }^{6,14,15}$ A recent international review of the incidence and prevalence of comorbidity in MS concluded that, despite growing interest in the impact of comorbidity on outcomes in MS, and the emergence of therapies that increase the risk of comorbidity or that may be relatively contraindicated in the presence of comorbidity, relatively little high-quality information on the prevalence of comorbidities in MS is available. ${ }^{13}$ Such information is especially important for patients with a dynamic disease like MS who have notable complexity due to the addition of multiple comorbidities.

There are a particularly limited number of published comorbidity studies in patients with MS in the US setting, ${ }^{5,16-22}$ and only one identified published study was a large population-based evaluation. Using data from the US Department of Defense database, Capkun et $a{ }^{16}$ compared mortality rates and event rates for comorbidities in MS $(n=15,684)$ and non-MS $(n=78,420)$ cohorts. The authors found that all-cause mortality was 2.9-fold higher in the MS versus non-MS cohort (mortality rate ratio, 95\% CI: 2.9, 2.7-3.2). Frequent causes of death in the MS versus non-MS cohort were infectious diseases $(6.2,4.2-9.4)$; diseases of the nervous (5.8, $3.7-9.0)$, respiratory $(5.0,3.9-6.4)$, and circulatory $(2.1$, 1.7-2.7) systems; and suicide $(2.6,1.3-5.2)$. The authors also found that the rates of several comorbidities were higher in patients with MS compared with patients without MS, including sepsis (event rate ratio, 95\% CI: 5.7, 5.1-6.3), ischemic stroke (3.8, 3.5-4.2), attempted suicide (2.4, 1.3-4.5), ulcerative colitis $(2.0,1.7-2.3)$, lymphoproliferative disorders $(2.2,1.9-2.6)$, and melanoma $(1.7,1.4-2.0){ }^{16}$

This study was performed to assess the trends in the prevalence of claims for comorbidities in US patients with MS by year from 2006 to 2014, and to determine the association of sex, age, and region with the presence of claims for comorbidities using a large US administrative claims database.

\section{Methods}

\section{Patient population}

This retrospective database analysis of patients with MS was conducted using data from a random sample of 5 million patients from the proprietary IMS Health Real World Data (RWD) Adjudicated Claims - US database. The IMS RWD Adjudicated Claims - US database is comprised of commercial health plan information from managed care plans throughout the US, with adjudicated claims of more than 150 million unique enrollees since 2006. This anonymous, patient-centric database includes all medical and pharmacy claims data, as well as demographic variables (age, sex, region of the US), eligibility by month, and the adjudicated payment for services. The database is de-identified and compliant with the Health Insurance Portability and Accountability Act of 1996. As such, no institutional review board approval was required.

\section{Analyses}

\section{Demographic characteristics}

Baseline demographics were evaluated for patients with MS in the dataset from 2006 to 2014. To be identified as having an MS diagnosis in a given year, a patient was required to have $\geq 2$ MS diagnosis claims (International Classification of Diseases, Ninth Revision, Clinical Modification [ICD-9-CM] code $=340 . x x$ ) at least 30 days apart (per Capkun et al, ${ }^{23}$ criteria for confirmed MS diagnosis), as well as continuous eligibility for at least 30 days. ${ }^{22}$ Patients could be included in multiple time periods (calendar years) if they met the eligibility criteria between January 1 and December 31 for any given year. Patient demographics that were assessed were age at start of 
the calendar year, age category $(<18,18-44,45-64$, or $\geq 65$ years), sex, and census region (Midwest, Northeast, South, or West). Census region was selected to assess geographic variation in health care encounters for comorbidities across the US.

\section{Assessment of comorbidities}

The comorbidities assessed were alcohol abuse, anxiety, arthritis (psoriasis, rheumatoid arthritis, or osteoarthritis), chronic lung disease, depression, diabetes (type I and type II), gastrointestinal disease (celiac disease, constipation, Crohn's disease, diarrhea, dysphagia, gastroesophageal reflux disease, irritable bowel syndrome, or ulcerative colitis), hyperlipidemia, hypertension, and thyroid disease. These comorbidities were selected as they are the most common among patients with MS based on a recent review of the published literature. ${ }^{11}$ A detailed list of the ICD-9 diagnosis codes used for the comorbidities can be obtained from the authors.

Rates of claims for comorbidities in patients with MS were calculated for each year from 2006 to 2014. Comorbidity claim rates were calculated by dividing the number of patients with $\geq 1$ month eligibility during the respective year, $\geq 2$ medical claims at any service location with an MS diagnosis at least 30 days apart, and $\geq 1$ medical claim for the comorbidity of interest by the number of patients with $\geq 1$ month eligibility during the respective year and $\geq 2$ medical claims at any service location with an MS diagnosis at least 30 days apart.

\section{Statistical analyses}

All descriptive analyses included mean and SD values for continuous measures, as well as proportions for binary and categorical measures. Factors associated with the presence of a given comorbidity were evaluated using logistic regression.
Variables investigated were age category (18-34, 35-44, $45-54,55-64$, and $\geq 65$ years), sex, and geographic region of the US (census region). Separate regressions were conducted for each included comorbidity. Although patients could be included for multiple years of evaluation for the annual analyses, the logistic regression used one record for each patient (ie, any data available for the patient from 2006-2014).

\section{Results \\ Demographic characteristics}

The number of patients in the individual annual cohorts ranged from 23,695 to 35,732 . The mean age of patients with MS from 2006-2014 ranged from 46.7-47.8 years old. Most patients with MS were between 45-54 years of age (31.7\%-35.2\%), 35-44 years of age $(23.3 \%-25.6 \%)$, or 55-64 years of age $(21.8 \%-27.3 \%)$. Approximately three quarters of patients were female (range of $76.2 \%-77.1 \%$ ). Patients with MS in this database were more likely to reside in the Northeast $(28.7 \%-37.4 \%)$ or Midwest $(28.6 \%-33.9 \%)$.

\section{Rates of comorbidities}

The most common comorbidities from 2006 to 2014 were hyperlipidemia and hypertension (25.9\%-29.7\% of patients within an individual year), followed by gastrointestinal disease (18.4\%-21.2\% of patients) and thyroid disease (12.9\%-17.1\% of patients). Chronic lung disease, arthritis, anxiety, diabetes, and depression were present in approximately $5 \%-10 \%$ of patients. A diagnosis of alcohol abuse was not commonly identified as a comorbidity in the claims dataset $(<1 \%)$ and is likely significantly underestimated as medical claims may not be commonly associated with this diagnosis (Figure 1).

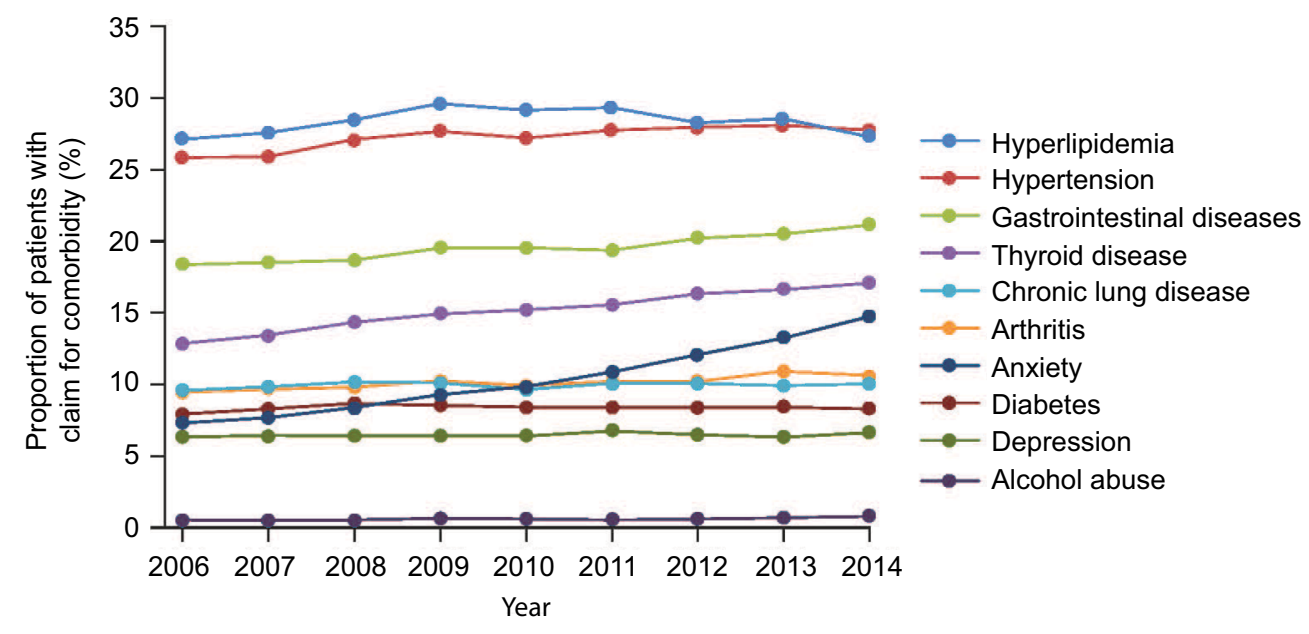

Figure I Overall rates of comorbidity claims for patients with MS from 2006-20I4. Abbreviation: MS, multiple sclerosis. 
The proportion of patients with a claim for hyperlipidemia increased from 2006 to 2009, was generally stable from 2009 to 2011, and then declined from 2011 to 2014. The proportion of patients with a claim for hypertension generally increased from 2006 to 2013, and then declined from 2013 to 2014. The proportion of patients with MS with a claim for gastrointestinal disease, thyroid disease, and anxiety generally increased from 2006 to 2014 (Figure 1).

Logistic regression models evaluating the impact of age, sex, and region found that claims for all comorbidities were statistically significantly more likely among older age groups $(p<0.05)$, with the exception of anxiety and alcohol abuse, which were statistically significantly less likely among older age groups (Table 1). Claims for gastrointestinal disease $(\mathrm{OR}=0.75)$, thyroid disease $(\mathrm{OR}=0.36)$, chronic lung disease $(\mathrm{OR}=0.76)$, arthritis $(\mathrm{OR}=0.71)$, anxiety $(\mathrm{OR}=0.63)$, and depression $(\mathrm{OR}=0.69)$ were statistically significantly less likely among males versus females (all $p<0.05$ ). Claims for hyperlipidemia $(\mathrm{OR}=1.39)$, hypertension $(\mathrm{OR}=1.25)$, diabetes $(\mathrm{OR}=1.31)$, and alcohol abuse $(\mathrm{OR}=2.41)$ were statistically significantly more likely among male patients compared with female patients with MS (Table 1). Patients in the Northeast had a statistically significantly higher likelihood of diagnosis claims of all the comorbidities compared with patients in the Midwest $(p<0.05)$ except for diabetes, depression, and alcohol abuse. Patients in the South had a statistically significantly higher likelihood of diagnosis claims for hyperlipidemia, hypertension, gastrointestinal disease, thyroid disease, and arthritis, anxiety, and diabetes, and a statistically significantly lower likelihood of depression and alcohol abuse compared with patients in the Midwest $(p<0.05)$. Patients in the West had a statistically significantly lower likelihood of diagnosis claims of all the comorbidities compared with patients in the Midwest $(p<0.05)$ except for thyroid disease and alcohol abuse (Table 1).

\section{Discussion}

A systematic review of the prevalence of comorbidity in MS identified substantial gaps in the epidemiological knowledge of comorbidity in MS worldwide. ${ }^{6}$ Due to heterogeneous study populations and designs, reported prevalence of comorbidities in MS varies widely, and only a small number of studies are population-based. ${ }^{6}$ Very limited data are available for the US setting of care. ${ }^{5,16-22}$ Only one of the published studies is population-based, ${ }^{16}$ and most of the studies are based on patient-reported estimates of comorbidity., ${ }^{5,19-21}$ Administrative claims data are of interest as a potential resource for population-based data analyses and for ongoing surveillance of comorbidities in MS. ${ }^{6,24-26}$

Comorbidity results observed in this study are consistent with the published literature for other countries. Hypertension and hyperlipidemia are among the most common comorbidities in MS (present in more than $25 \%$ of patients), thyroid disease is one of the most prevalent comorbid autoimmune diseases, and chronic lung disease, arthritis, and diabetes occur in approximately $5 \%-10 \%$ of patients with MS. ${ }^{6}$ The rates of diabetes, hypertension, and hyperlipidemia are significant in the MS population. ${ }^{27}$ These vascular comorbidities are particularly noteworthy as they have been shown to be associated with increased disability progression in MS. ${ }^{8} \mathrm{~A}$ single vascular comorbidity at diagnosis was associated with a $51 \%$ increased risk of early gait disability, while two vascular comorbidities was associated with a $228 \%$ increased risk. $^{8}$

In regards to the trends in comorbidity claims in this study, it was interesting to note that the proportion of patients with MS with a claim for gastrointestinal disease, thyroid disease,

Table I Logistic regression models of comorbidities adjusting for age, sex, and region for patients with MS

\begin{tabular}{|c|c|c|c|c|c|c|c|c|}
\hline \multicolumn{9}{|c|}{ Odds ratios } \\
\hline & & \multicolumn{4}{|c|}{ Age ( $<34$ years reference) } & \multicolumn{3}{|c|}{ Region (Midwest reference) } \\
\hline & \begin{tabular}{|l|} 
Sex (female \\
reference)
\end{tabular} & $35-44$ & $45-54$ & $55-64$ & $\geq 65$ & Northeast & South & West \\
\hline Hyperlipidemia & 1.39 & 2.60 & 5.57 & 10.16 & 11.28 & 1.26 & 1.25 & 0.82 \\
\hline Hypertension & 1.25 & 2.42 & 5.23 & 9.81 & 18.39 & 1.11 & 1.33 & 0.77 \\
\hline Gastrointestinal disease & 0.75 & 1.15 & 1.53 & 2.02 & 2.93 & 1.08 & 1.17 & 0.93 \\
\hline Thyroid disease & 0.36 & 1.54 & 2.06 & 2.64 & 3.08 & 1.14 & 1.17 & $1.02^{\mathrm{a}}$ \\
\hline Chronic lung disease & 0.76 & $1.00^{\mathrm{a}}$ & 1.15 & 1.43 & 2.36 & 1.08 & $1.02^{\mathrm{a}}$ & 0.86 \\
\hline Arthritis & 0.71 & 2.08 & 4.13 & 6.94 & 10.69 & 1.10 & 1.09 & 0.90 \\
\hline Anxiety & 0.63 & 0.88 & 0.73 & 0.63 & 0.52 & 1.10 & 1.12 & 0.87 \\
\hline Diabetes & 1.31 & 1.85 & 3.10 & 5.09 & 7.77 & 0.96 & 1.07 & 0.80 \\
\hline Depression & 0.69 & 1.22 & 1.18 & 1.13 & 0.90 & 0.87 & 0.68 & 0.80 \\
\hline Alcohol abuse & 2.41 & $0.93^{\mathrm{a}}$ & 0.86 & 0.59 & 0.43 & $0.98^{\mathrm{a}}$ & 0.76 & $0.96^{a}$ \\
\hline
\end{tabular}

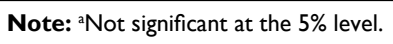

Abbreviation: MS, multiple sclerosis. 
and anxiety have generally been increasing over time. The proportion of patients with a claim for hyperlipidemia and hypertension was also increasing, but may have stabilized or may be declining. The proportion of patients with a claim for hypertension generally increased from 2006 to 2013, and then declined from 2013 to 2014. Possible reasons for these shifts may be better preventive health care over time, or changes in the population of patients with MS such that patients with MS interacting with the health care system in recent years have better cardiovascular health but are experiencing greater stress. These would be interesting areas for future research.

The present study demonstrates the importance of considering population-based characteristics when evaluating comorbidities in MS as prevalence may vary based on age, sex, and geographic region of patients. It is important for health care providers to understand which comorbidities may be more or less prevalent in certain demographic groups to improve available clinical support, health care services, and quality of life for these patients. Managed care may also be able to use these findings to identify patients who may benefit from interventions. Some patients with MS may not be receiving the recommended preventive care services, ${ }^{28}$ and some of the comorbid conditions prevalent in patients with MS may be prevented with proper screenings or better managed with additional patient counseling. ${ }^{29}$ Care of patients with MS is optimized with a comprehensive care approach, ${ }^{30}$ and the increased complexity from comorbidities reinforces the importance of integrated care.

There are some limitations associated with this study. The ICD-9-CM code for systemic MS does not distinguish between different MS types (eg, primary progressive MS, relapsingremitting MS, or secondary progressive MS). Also, claims data are subject to possible coding errors and undercoding. ${ }^{31}$ The use of administrative claims data for obtaining information about comorbidities has limitations in that claims data are not collected for research purposes, but rather for reimbursement information (claims processing and payment). ${ }^{31}$ For example, the diagnosis criteria for alcohol abuse and mental illness are more subjective than for other medical conditions and can lead to undercoding in administrative claims data. ${ }^{32}$ Furthermore, services may not be captured in the claims database because the particular service is not covered by the plan sponsor or because the service is 'carved-out' and not captured in the data set (eg, a mental health condition may not be addressed during a particular service and thus may not be captured in the claim). ${ }^{31}$ Data fields that are not required for reimbursement may be particularly unreliable. ${ }^{31}$

The use of a single encounter for evaluating the annual rate of comorbidity claims in this study may not be adequate for confirming the presence of comorbidities. The focus of our study was to evaluate claims for common comorbidities over time and to determine how these health care encounters varied by age, sex, and geographic region. Also, other variables and potential confounders not available in the claims data may be responsible for the differences observed. Finally, administrative claims databases provide information on patients with health insurance administered by health plans in the US; therefore, results may not be generalizable to patients who self-pay or patients without employer-sponsored commercial health insurance. Further research in other populations is warranted.

The study was designed to provide an overview of comorbidities in US patients with MS, and it highlights the complexity of treatment of patients with MS. Some patients with MS and other types of chronic diseases and multiple comorbidities may be more likely to receive interventions such as medication therapy management to help with the simultaneous management of their medical conditions. ${ }^{33} \mathrm{By}$ sharing expertise in a coordinated and collaborative manner, the complex needs of individuals with MS and their caregivers may be better met. ${ }^{30}$

\section{Conclusion}

These analyses provide estimates of claims for comorbidities in US patients with MS and suggest that demographic characteristics are associated with comorbidities. Current and reliable estimates of the rates of comorbidities are essential in order to improve available clinical support, health care services, and quality of life for patients with MS. Comprehensive care for the MS patient and their comorbidities may produce better outcomes for patients and their caregivers. Claims data are an important source of information for studies of the burden of disease.

\section{Acknowledgments}

The study was supported by EMD Serono, Inc., Rockland, MA, USA (a business of Merck KGaA, Darmstadt, Germany). The authors thank Stacey Reeber, PhD, of Caudex, New York, NY (supported by EMD Serono, Inc., Rockland, MA, USA [a business of Merck KGaA, Darmstadt, Germany]) for editorial assistance in drafting the manuscript, collating the comments of authors, and assembling tables and figures.

\section{Disclosure}

NCE has received compensation as a Health Services Research Consultant. MM and JM received research support from EMD Serono, Inc., for the presented analysis. ALP is 
an employee of EMD Serono, Inc., Rockland, MA, USA (a business of Merck KGaA, Darmstadt, Germany). The authors report no other conflicts of interest in this work.

\section{References}

1. Luessi F, Siffrin V, Zipp F. Neurodegeneration in multiple sclerosis: novel treatment strategies. Expert Rev Neurother. 2012;12(9):1061-1076.

2. Dilokthornsakul P, Valuck RJ, Nair KV, Corboy JR, Allen RR, Campbell JD. Multiple sclerosis prevalence in the United States commercially insured population. Neurology. 2016;86(11):1014-1021.

3. nationalmssociety.org [homepage on the Internet]. MS Prevalence. National Multiple Sclerosis Society. Available from: http://www. nationalmssociety.org/About-the-Society/MS-Prevalence. Accessed January 25, 2018.

4. Multiple Sclerosis International Federation. Atlas of MS 2013. Multiple Sclerosis International Federation. Available from: http://www.msif.org/ wp-content/uploads/2014/09/Atlas-of-MS.pdf. Accessed January 25, 2018.

5. nationalmssociety.org [homepage on the Internet]. How Common Is It To Have MS With Other Medical Conditions? First Results from the MS “Comorbidities” Project. National Multiple Sclerosis Society; 2015. Available from: http://www.nationalmssociety.org/About-the-Society/ News/How-Common-Is-It-To-Have-MS-With-Other-Medical-Con. Accessed January 25, 2018.

6. Marrie R, Horwitz R, Cutter G, Tyry T, Campagnolo D, Vollmer T. Comorbidity, socioeconomic status and multiple sclerosis. Mult Scler. 2008;14(8):1091-1098.

7. Marrie RA, Horwitz R, Cutter G, Tyry T, Campagnolo D, Vollmer T. Comorbidity delays diagnosis and increases disability at diagnosis in MS. Neurology. 2009;72(2):117-124.

8. Marrie RA, Rudick R, Horwitz R, Cutter G, Tyry T, Campagnolo D, Vollmer T. Vascular comorbidity is associated with more rapid disability progression in multiple sclerosis. Neurology. 2010;74(13):1041-1047.

9. Berrigan LI, Fisk JD, Patten SB, et al. Health-related quality of life in multiple sclerosis: Direct and indirect effects of comorbidity. Neurology. Epub 2016 Mar 9.

10. Warren SA, Turpin KVL, Pohar SL, Jones CA, Warren KG. Comorbidity and health-related quality of life in people with multiple sclerosis. International Journal of MS Care. 2009;11(1):6-16.

11. Marrie RA, Elliott L, Marriott J, Cossoy M, Tennakoon A, Yu N. Comorbidity increases the risk of hospitalizations in multiple sclerosis. Neurology. 2015;84(4):350-358.

12. Marrie RA, Elliott L, Marriott J, Cossoy M, Blanchard J, Leung S, Yu N. Effect of comorbidity on mortality in multiple sclerosis. Neurology. 2015;85(3):240-247.

13. Marrie RA, Cohen J, Stuve O, et al. A systematic review of the incidence and prevalence of comorbidity in multiple sclerosis: overview. Mult Scler. 2015;21(3):263-281.

14. Marck CH, Neate SL, Taylor KL, Weiland TJ, Jelinek GA. Prevalence of comorbidities, overweight and obesity in an international sample of people with multiple sclerosis and associations with modifiable lifestyle factors. PLoS One. 2016;11(2):e0148573.

15. Marrie RA, Miller A, Sormani MP, et al. Recommendations for observational studies of comorbidity in multiple sclerosis. Neurology. 2016;86(15):1446-1453.
16. Capkun G, Dahlke F, Lahoz R, et al. Mortality and comorbidities in patients with multiple sclerosis compared with a population without multiple sclerosis: An observational study using the US Department of Defense administrative claims database. Mult Scler Relat Disord. 2015;4(6):546-554.

17. Coyle CP, Santiago MC, Shank JW, Ma GX, Boyd R. Secondary conditions and women with physical disabilities: a descriptive study. Arch Phys Med Rehabil. 2000;81(10):1380-1387.

18. Fleming ST, Blake RL Jr. Patterns of comorbidity in elderly patients with multiple sclerosis. J Clin Epidemiol. 1994;47(10):1127-1132.

19. Marrie RA, Horwitz RI, Cutter G, Tyry T, Vollmer T. Association between comorbidity and clinical characteristics of MS. Acta Neurol Scand. 2011;124(2):135-141.

20. Motl RW, Gappmaier E, Nelson K, Benedict RH. Physical activity and cognitive function in multiple sclerosis. J Sport Exerc Psychol. 2011;33(5):734-741.

21. Newland P, Jensen MP, Budhathoki C, Lorenz R. Secondary health conditions in individuals with multiple sclerosis: a cross-sectional web-based survey analysis. J Neurosci Nurs. 2015;47(3):124-130.

22. Redelings MD, McCoy L, Sorvillo F. Multiple sclerosis mortality and patterns of comorbidity in the United States from 1990 to 2001. Neuroepidemiology. 2006;26(2):102-107.

23. Capkun G, Lahoz R, Verdun E, et al. Expanding the use of administrative claims databases in conducting clinical real-world evidence studies in multiple sclerosis. Curr Med Res Opin. 2015;31(5):1029-1039.

24. Marrie RA, Yu BN, Leung S, et al. Rising prevalence of vascular comorbidities in multiple sclerosis: validation of administrative definitions for diabetes, hypertension, and hyperlipidemia. Mult Scler. 2012;18(9):1310-1319.

25. Marrie RA, Fisk JD, Yu BN, et al. Mental comorbidity and multiple sclerosis: validating administrative data to support population-based surveillance. BMC Neurol. 2013;13:16.

26. Marrie RA, Yu BN, Leung S, et al. The utility of administrative data for surveillance of comorbidity in multiple sclerosis: a validation study. Neuroepidemiology. 2013;40(2):85-92.

27. Marrie RA, Fisk J, Tremlett H, et al. Differing trends in the incidence of vascular comorbidity in MS and the general population. Neurol Clin Pract. 2016;6(2):120-128.

28. Barrett MW, Roberts B. Preventive screening in people with multiple sclerosis. International Journal of MS Care. 2010;12:168-176.

29. uspreventiveservicestaskforce.org [homepage on the Internet]. About the USPSTF. U.S. Preventive Services Task Force; 2017. Available from: https://www.uspreventiveservicestaskforce.org/Page/Name/about-theuspstf. Accessed January 25, 2018.

30. nationalmssociety.org [homepage on the Internet]. Comprehensive Care. National Multiple Sclerosis Society. Available from: https://www. nationalmssociety.org/Treating-MS/Comprehensive-Care. Accessed January 25, 2018.

31. Motheral B, Brooks J, Clark MA, et al. A checklist for retrospective database studies--report of the ISPOR Task Force on Retrospective Databases. Value Health. 2003;6(2):90-97.

32. Kim HM, Smith EG, Stano CM, Ganoczy D, Zivin K, Walters H, Valenstein M. Validation of key behaviourally based mental health diagnoses in administrative data: suicide attempt, alcohol abuse, illicit drug abuse and tobacco use. BMC Health Serv Res. 2012;12:18.

33. Anderson SS, Philbrick AM. Improving multiple sclerosis care: an analysis of the necessity for medication therapy management services among the patient population. J Manag Care Spec Pharm. 2014;20(3):254-261.
Patient Related Outcome Measures

\section{Publish your work in this journal}

Patient Related Outcome Measures is an international, peer-reviewed, open access journal focusing on treatment outcomes specifically relevant to patients. All aspects of patient care are addressed within the journal and practitioners from all disciplines are invited to submit their work as well as healthcare researchers and patient support groups.

\section{Dovepress}

The journal is included in PubMed. The manuscript management system is completely online and includes a very quick and fair peer-review system. Visit http://www.dovepress.com/testimonials.php to read real quotes from published authors. 